\title{
Metabolic Disease Risk Alters Circulating Peripheral Blood Mononuclear Cell microRNAs in Response to A High Glycemic Meal ${ }^{\dagger}$
}

\author{
Farha Ramzan 1,2, Randall F. D'Souza 1, Brenan R. Durainayagam ', Amber M. Milan 1, \\ James F. Markworth ${ }^{1}$, Nicole C. Roy ${ }^{2,3,4}$, Cameron J. Mitchell ${ }^{1}$ and David Cameron-Smith ${ }^{1,2,3,5, *}$ \\ 1 Liggins Institute, The University of Auckland, Auckland 1023, New Zealand; \\ f.ramzan@auckland.ac.nz (F.R.); r.dsouza@auckland.ac.nz (R.F.D.); \\ b.durainayagam@auckland.ac.nz (B.R.D.); a.milan@auckland.ac.nz (A.M.M.); \\ jmarkwor@med.umich.edu (J.F.M.); cameron.mitchell@ubc.ca (C.J.M.) \\ 2 Riddet Institute, Palmerston North, 4442, New Zealand; nicole.roy@agresearch.co.nz \\ 3 Food Nutrition \& Health Team, AgResearch Limited, Palmerston North 4442, New Zealand \\ 4 High-Value Nutrition National Science Challenge, Auckland 1023, New Zealand \\ 5 Food \& Bio-based Products Group, AgResearch Limited, Palmerston North 4442, New Zealand \\ * Correspondence: d.cameron-smith@auckland.ac.nz; Tel.: +64-9-923-1336 \\ † Presented at the 2018 Nutrition Society of New Zealand Annual Conference, Auckland, New Zealand, \\ 28-30 November 2018.
}

Published: 12 March 2019

Background: High glycemic diets have been shown to exacerbate the risk of cardio-metabolic disease in individuals with pre-existing disease risk, including obesity and insulin resistance, common to the Metabolic Syndrome (MetS). Whilst the dysregulation of microRNAs (miRNAs) has been associated with the molecular events underpinning cardio-metabolic risk, little is understood about their dietary regulation. Therefore, we aimed to identify alterations in the expression of peripheral blood mononuclear cell (PBMC) miRNAs mediated by acute high glycemic meal intake in individuals with or without metabolic syndrome (MetS).

Methods: 40 female (20 healthy [63 \pm 1.0 years] and $20 \mathrm{MetS}$ [ $62.6 \pm 1.2$ years]) participants were fed a high glycemic meal following an overnight fast. PBMCs were isolated from blood samples collected at baseline as well as 2 and $4 \mathrm{~h}$ post meal. miRNA expression was determined using qPCR. In silico pathways analysis utilising the Kyoto Encyclopedia of Genes and Genomes (KEGG) was performed to identify putative affected gene networks.

Results: Of the 12 miRNAs analysed, two (miR-126-3p, and miR-451a) showed differential expression between MetS and healthy subjects at baseline. In MetS subjects, miR-16-5p and -451a showed a significant downregulation $4 \mathrm{~h}$ post meal $(p<0.05)$, whilst in healthy subjects, miR-320a showed downregulated expression $4 \mathrm{~h}$ post meal. In silico analysis showed miR-16-5p targeting the highest number of genes, with its downregulation leading to predicted upregulated expression of target genes in pathways including MAPK, ErbB, VEGF, and insulin signaling.

Conclusions: A high glycemic meal leads to altered expression of miRNAs in circulating PBMCs. The response was different between MetS and healthy subjects, with a predominant reduction of miRNAs that regulate inflammatory and metabolic pathway gene expression. This study provides insights for future studies to confirm the role of altered miRNAs.

Supplementary Materials: The poster is available online at www.mdpi.com/2504-3900/8/1/30/s1. 